\title{
Análise dos Efeitos de Histórias de Variação Comportamental sobre o Seguimento de Regras
}

\author{
José Guilherme Wady Santos ${ }^{1}$ \\ Carla Cristina Paiva Paracampo ${ }^{2}$ \\ Luiz Carlos de Albuquerque \\ Universidade Federal do Pará
}

\begin{abstract}
Resumo
Investigando a sensibilidade do seguir instruções à mudança (sinalizada) nas contingências, 14 crianças, entre $8 \mathrm{e} 9$ anos de idade, foram expostas a um procedimento de escolha segundo o modelo. A tarefa era tocar 1 dos 2 estímulos de comparação na presença de 1 estímulo contextual. $O$ experimento consistia de 3 fases; as contingências em vigor na Fase 1 eram revertidas na Fase 2 e restabelecidas na Fase 3 . Transições de uma fase para outra eram sinalizadas. Os participantes foram atribuídos a 2 condições, que diferiam quanto ao número de instruções, correspondentes às contingências, apresentadas na Fase 1: era apresentada 1 única instrução na Condição UI e 3 diferentes instruções na Condição MI. Observou-se que 1 dos 6 participantes da Condição UI e 4 dos 8 da Condição MI deixaram de seguir instruções. Sugere-se que a interação entre a história de variação comportamental, gerada por diferentes instruções, e a sinalização da mudança nas contingências, pode tornar o comportamento instruído sensível a tal mudança.

Palavras-chave: Comportamento governado por regras; comportamento verbal; variação comportamental; procedimento de escolha segundo o modelo; crianças.
\end{abstract}

An Analysis of the Effects of History of Behavioral Variation on Rule-Following Behavior

\begin{abstract}
In a study concerning the sensitivity of instruction-following to signaled changes in contingencies, 14 children in the 8-to- 9 age range were exposed to a matching-to-sample procedure. On this task, 1 of 2 comparison stimuli were touched in the presence of a contextual stimuli. There were 3 phases in the experiment. Those contingencies in effect in Phase 1 were reversed in Phase 2, and reestablished in Phase 3. The transition from one phase to another was cued by a signal. The participants were subjected to 2 conditions that differed in the number of instructions corresponding to the contingencies presented in Phase 1: Only 1 instruction was given in Condition UI, and 3 different instructions were given in Condition MI. One of the 6 participants in Condition UI and 4 of the 8 in Condition MI ceased following instructions. These results suggest that the presence of an interaction between history of behavioral variation produced by different instructions and signalling contingency shifts may render the ins tructed behavior susceptible to contingency shifts.

Keywords: Rule-governed behavior; verbal behavior; behavioral variation; matching-to-sample; children.
\end{abstract}

De acordo com Skinner (1969), regras são estímulos especificadores de contingências e exercem controle como estímulos discriminativos, fazendo parte de um conjunto de contingências de reforço. Ou seja, são estímulos que podem especificar o comportamento a ser emitido (a forma, a freqüência e a duração do comportamento), as condições sob as quais ele deve ser emitido (quando e onde o comportamento deve ocorrer), e suas prováveis conseqüências (o que poderá acontecer se a regra for seguida). Por esta definição ${ }^{3}$, instruções, avisos, conselhos, ordens, leis seriam exemplos particulares de regras, uma vez que todos podem descrever contingências e funcionar como estímulos discriminativos (Albuquerque \& Ferreira, 2001).

\footnotetext{
${ }^{1}$ Este trabalho foi baseado nos dados da dissertação de mestrado do primeiro autor, apresentada em 2002 no Programa de Pós-Graduação em Teoria e Pesquisa do Comportamento/UFPA, e foi realizado com o auxílio financeiro da CAPES em forma de bolsa concedida ao primeiro autor.

${ }^{2}$ Endereço para correspondência: Rua Oliveira Belo, 238,1702, 66050 380, Umarizal, Belém, PA. Fone: (91) 2238995. E-mail:ccpp@cpgp.ufpa.br

${ }^{3}$ No presente estudo o termo "regra" está sendo usado em situações mais gerais e o termo "instrução" está sendo usado em situações mais particulares, como nas descrições dos procedimentos e resultados de pesquisas.
}

Ainda de acordo com Skinner (1969), o comportamento governado por regras e o comportamento modelado por contingências podem ter topografias similares, mas as suas variáveis de controle são distintas; portanto, são operantes distintos. Mais especificamente, pode-se dizer que um determinado comportamento particular é controlado por contingências quando o comportamento é estabelecido por suas conseqüências imediatas, enão por uma regra. Pode ser classificado como controlado por regras quando o comportamento que se segue à apresentação de uma regra é o previamente especificado pela regra e este comportamento ocorre independentemente de suas conseqüências imediatas. E pode ainda ser classificado de controlado pela interação entre regra e contingência, quando é estabelecido por uma regra e sua manutenção depende de suas conseqüências imediatas (Albuquerque, 2001, 2002; Albuquerque, de Souza, Matos \& Paracampo, 2003; Albuquerque, Matos, de Souza \& Paracampo, no prelo). Assim, as conseqüências responsáveis pela manutenção do comportamento governado por regras, enquanto um operante de ordem superior, cujos membros seriam exemplos individuais de seguimento de regras particulares, seriam as conseqüências sociais para o responder de acordo com regras (Catania, Matthews \& Shimoff, 1990; Cerutti, 
1989). Conseqüências atuais produzidas porocorrências individuais de seguimento de regras particulares seriam conseqüências que poderiam alterar a probabilidade de certos comportamentos de seguir regras virem a ocorrer no futuro (Malott, 1989; Perone, Galizio \& Baron, 1988), mas não a sua probabilidade presente. A probabilidade presente seria determinada pela história do ouvinte (Hayes, Zettle \& Rosenfarb, 1989; Parrott, 1987).

Uma importante função de regras é simplificar as contingências de reforço no estabelecimento de um novo comportamento (Catania, 1999; Skinner, 1974). Regras podem simplificar as contingências de reforço, principalmente quando estas contingências são complexas, pouco claras, atuam apenas a longo prazo, ou mesmo são pouco eficazes. Regras também têm o efeito de ampliar o repertório dos indivíduos, uma vez que, ao descreverem as contingências de reforço, permitem aos mesmos entrarem em contato com contingências que talvez nunca fossem contatadas naturalmente. Um problema, no entanto, é que quando as contingências mudam e não as regras, estas poderão mais atrapalhar do que ajudar (Skinner, 1969).

A partir dessas proposições iniciais de Skinner, vários autores (Baron, Kaufman \& Stauber, 1969; Cerutti, 1991, 1994; Catania, Matthews \& Shimoff, 1982; Galizio, 1979; Hayes, Brownstein, Haas \& Greenway, 1986a; Hayes, Brownstein, Zettle, Rosenfarb \& Korn, 1986b; Joyce \& Chase, 1990; LeFrancois, Chase \& Joyce, 1988; Lippman \& Meyer, 1967; Lowe, 1979; Michael \& Bernstein, 1991; Paracampo, Albuquerque \& Fontes, 1993; Shimoff, Catania \& Matthews, 1981;Shimoff,Matthews \& Catania, 1986; Torgrud \& Holborn, 1990; Weiner, 1970), na análise experimental do comportamento, começaram a comparar o controle por regras com o controle por contingências sobre o comportamento humano, em esquemas de reforço.

Por exemplo, Lippman e Meyer (1967) expuseram humanos adultos a um esquema de FI $20 \mathrm{~s}$ e observaram que os participantes que haviam sido expostos a instruções que especificavam que o reforço estaria disponível de acordo com um esquema de FI, apresentaram baixa taxa de respostas e a curva típica de FI. Já os participantes que foram expostos a instruções que especificavam que o reforço estaria disponível de acordo com um esquema de razão, apresentaram um padrão de taxa alta de respostas; e os participantes que foram expostos apenas a instruções mínimas apresentaram ou um padrão de taxa alta ou um padrão de taxa baixa de respostas.

Weiner (1970) encontrou resultados similares quando expôs humanos adultos a um esquema de FR 10, no qual podiam receber até 700 reforços, seguido de 2 horas de extinção. Os participantes que não foram instruídos sobre o número de reforços que seriam apresentados no experimento, mostraram pouca tendência a parar de responder em extinção. Os participantes que foram expostos a instruções que especificavam que poderiam ganhar até 700 reforços, pararam de responder abruptamente naquele ponto ou emitiram poucas respostas em extinção. Já os participantes que foram expostos a instruções que especificavam que 999 reforços estariam disponíveis, apresentaram taxas altas de respostas durante a extinção.

Shimoff e colaboradores (1981) também encontraram resultados similares quando expuseram dois grupos de estudantes a um esquema de intervalo randômico (RI) superposto a um esquema de DRL, que posteriormente era descontinuado ao longo da sessão. Para o Grupo 1, o responder foi inicialmente estabelecido por modelagem e para o Grupo 2 foi estabelecido por instruções. Os resultados mostraram que quando a contingência de DRL foi descontinuada a taxa de respostas dos participantes do Grupo 1 aumentou, entretanto, não foram observadas mudanças sistemáticas na taxa de respostas dos participantes do Grupo 2.

Os resultados destes estudos sugerem que regras podem interferir na adaptação do comportamento não-verbal humano às contingências de reforço. Regras que descrevem as contingências de reforço podem facilitar a adaptação do comportamento às mesmas (Baron \& Galizio, 1983; Lippman \& Meyer, 1967; Weiner, 1970). No entanto, quando as contingências mudam, tornando as regras discrepantes das contingências, o comportamento estabelecido por regras tem menor probabilidade de mudar acompanhando tais mudanças do que o comportamento inicialmente estabelecido por modelagem ou reforço diferencial, ou seja, o comportamento estabelecido por regras parece pouco sensível ${ }^{4}$ a alterações nas contingências de reforço (Matthews, Shimoff, Catania \& Sagvolden, 1977; Shimoff \& cols., 1981).

Uma explicação para a freqüente insensibilidade do comportamento humano às contingências de reforço programadas em situações experimentais pode estar na maneira como regras e contingências restringem a variabilidade do comportamento. Ou seja, o comportamento governado por regras geralmente não apresenta variação em relação à regra. A topografia da resposta, na maioria das vezes, está descrita na regra e o indivíduo emite a resposta antes mesmo que as suas conseqüências imediatas possam exercer algum efeito sobre a mesma; isto é, no comportamento governado por regras os padrões de respostas são produzidos sem exposição direta às contingências de reforço descritas pelas regras. Em contraste, o comportamento modelado por contingências pode inicialmente apresentar grande variabilidade e uma longa exposição às contingências de reforço pode ser necessária antes que um padrão de respostas desejado seja selecionado (Joyce \& Chase, 1990).

Por esta proposição, para tornar-se sensível às contingências de reforço programadas, o comportamento deve ser exposto a condições que possam gerar variação comportamental antes ou

\footnotetext{
${ }^{4} \mathrm{O}$ termo sensibilidade tem sido usado para descrever o comportamento que está sob controle de suas conseqüências imediatas e o termo insensibilidade para descrever o comportamento que não está sob controle de suas conseqüências imediatas em uma determinada situação particular.
} 
no momento das mudanças nessas contingências (Chase \& Danforth, 1991; Joyce \& Chase, 1990; LeFrancois \& cols., 1988).

Por exemplo, LeFrancois e colaboradores (1988) estudaram o efeito da exposição prévia a diferentes instruções relativas a diferentes esquemas de reforço sobre a sensibilidade do desempenho após a mudança nas contingências. Para tanto, expuseram 90 estudantes a uma de seis condições experimentais. Cada condição era constituída por três fases: Fase de treino, que durava 32 minutos; Fase de teste, que durava 25 minutos e Fase de extinção, que durava 10 minutos. Nas Condições 1 e 2 a Fase de treino consistiu na apresentação de oito diferentes esquemas de reforço (FR 60, FR 100, DRL 15s, DRL 4s, FT 15s, FT 45s, VI 20s e VI 40s, na Condição 1; e FR 40, FR 60, FR 100, FT15s, FT 45s, VI 20s, VI 40s e VI 60s, na Condição 2), durante 4 minutos cada um. A apresentação de cada esquema era precedida pela apresentação, na tela do computador, de uma instrução correspondente ao esquema em efeito. Nas Condições 3 e 4 a Fase de treino consistiu na apresentação de um único esquema de reforço (VI 30s na Condição 3 e VR 80 na Condição 4) precedida pela apresentação de uma instrução correspondente ao esquema em efeito. Nas Condições 5 e 6 a Fase de treino também consistiu na apresentação de um único esquema de reforço (VI 30s na Condição 5 e VR 80 na Condição 6), só que precedida pela apresentação de instruções mínimas, que não especificavam o padrão de respostas que produzia pontos. Em todas as condições, o início da Fase de teste foi precedido pela apresentação de uma instrução que dizia para o participante descobrir qual a melhor maneira de ganhar pontos. Em seguida, os participantes eram expostos a um esquema de FI 30s. Após a Fase de teste, sem a apresentação de qualquer sinalização, os participantes de todas as condições foram expostos a uma Fase de Extinção.

Os resultados mostraram que o desempenho de 25 dos 30 participantes das Condições 1 e 2 mudou (da Fase de treino para a Fase de teste) acompanhando a mudança nas contingências de reforço programadas. Essa mudança ocorreu com o desempenho de apenas 14 dos 60 participantes das outras quatro condições. Em outras palavras, a maioria dos participantes das Condições 1 e 2 mudou as taxas e o padrão de respostas da Fase de treino para a Fase de teste. Já a maioria dos participantes das Condições 4, 5 e 6 continuou apresentando, nas Fases de teste e extinção, as mesmas taxas altas de respostas apresentadas na Fase de treino. A maioria dos participantes da Condição 3 manteve-se apresentando, nas Fases de teste e extinção, as mesmas taxas baixas de respostas apresentadas na Fase de treino.

De acordo com LeFrancois e colaboradores (1988), estes resultados sugerem que é mais provável que o comportamento humano mude acompanhando a mudança nas contingências de reforço quando é previamente exposto a diferentes instruções, sobre diferentes esquemas de reforço apresentados sucessivamente, do que quando é exposto a apenas uma instrução relativa a um único esquema. Uma possível razão para isso pode estar no fato de o treino ter envolvido mudanças no padrão das respostas. Dessa forma, quando confrontado com um novo esquema, o padrão de respostas mudou.

Nessa mesma linha de pesquisa, Joyce e Chase (1990) investigaram se instruções para variar entre taxas altas e baixas gerariam desempenho sensível à mudança nas contingências. Seis estudantes universitários foram distribuídos em duas condições experimentais. Em ambas as condições os participantes foram inicialmente expostos a um esquema de FR 40. Na Condição 1 foram expostos a instruções correspondentes que especificavam que deveriam pressionar um botão 40 vezes para obter pontos trocáveis por dinheiro. $\mathrm{Na}$ Condição 2 foram expostos a instruções mínimas que especificavam apenas que pontos poderiam ser obtidos pressionando-se o botão. Após o desempenho de todos eles atingirem um critério de estabilidade em FR 40, os participantes foram expostos a um teste de sensibilidade que consistiu na apresentação do esquema de FR 40, até serem obtidos seis pontos, seguida da apresentação de um esquema de FI 10 s, por 15 minutos. Depois, os participantes foram expostos a instruções para variar entre taxas altas e baixas de respostas e, em seguida, eram novamente expostos às mesmas contingências do teste de sensibilidade.

Os resultados mostraram que todos os participantes das duas condições apresentaram taxas altas de respostas durante o teste de sensibilidade. Após a apresentação das instruções para variar, passaram a apresentar um padrão de respostas variável, caracterizado pela alternação entre taxas altas e baixas de respostas. Com o decorrer da sessão passaram a apresentar um desempenho sob controle das contingências de FI. Os autores concluíram que instruções para variar podem gerar desempenho sensível à mudança nas contingências. Ou seja, de acordo com os autores, se variação comportamental gerada por instruções produzir respostas alternativas que mantenham contato com as contingências de reforço, estas contingências poderiam selecionar estas respostas alternativas e, neste caso, o comportamento seria sensivel às contingências.

Ainda nesta mesma linha de investigação, Paracampo, Souza, Matos e Albuquerque (2001) procuraram avaliar os efeitos, sobre o comportamento verbal e o não-verbal, de mudanças nas contingências de reforço programadas para o comportamento não-verbal. Para tanto, expuseram 20 crianças, entre 7 e 8 anos de idade, a um procedimento de controle contextual de escolha segundo o modelo. $\mathrm{O}$ experimento era constituído de três condições e, cada condição, de três fases. As condições diferiram quanto às instruções apresentadas no início da Fase 1. Na Condição Reforço Diferencial (RD), os participantes foram expostos a instruções mínimas sobre como proceder; na Condição Instrução (CI) foram expostos a instruções correspondentes às contingências em vigor na Fase 1; e na Condição Múltiplas Instruções (MI) foram expostos a três conjuntos de instruções correspondentes (Passos 1, 2 e 3), de 
acordo com três mudanças nas contingências em vigor na Fase 1. $\mathrm{Na}$ Fase 1 das Condições $\mathrm{RD} \mathrm{e} \mathrm{CI} \mathrm{eram} \mathrm{reforçadas} \mathrm{as} \mathrm{respostas}$ de escolher o estímulo de comparação igual ao modelo na presença da luz verde e o diferente do modelo na presença da luz vermelha. Estas contingências em vigor na Fase 1 eram revertidas na Fase 2 e restabelecidas na Fase 3. Na Fase 1 da Condição MI eram reforçadas as respostas de escolher o igual na presença da luz verde e o diferente na presença da luz amarela no Passo 1, e escolher o igual na presença da luz amarela e o diferente na presença da luz vermelha no Passo 2, e escolher igual na presença da luz verde e o diferente na presença da luz vermelha no Passo 3. Estas contingências em vigor no Passo 3 eram revertidas na Fase 2 e restabelecidas na Fase 3. Transições de uma fase para outra não eram sinalizadas e nem instruídas. Durante cada fase os participantes eram indagados sobre o que deveriam fazer para ganhar fichas. As respostas não-verbais de acordo com as contingências eram reforçadas em esquema de reforço contínuo e as respostas verbais não eram reforçadas diferencialmente.

Os resultados mostraram que nas três condições o comportamento verbal sempre correspondeu ao não-verbal em todas as fases. $\mathrm{Na}$ Condição $\mathrm{RD}$, tanto o comportamento nãoverbal quanto o verbal mudaram acompanhando a mudança nas contingências de reforço. Para todos os participantes da Condição CI e para cinco dos seis participantes da Condição MI, tanto o comportamento verbal quanto o não-verbal permaneceram inalterados quando ocorreu mudança nas contingências de reforço. Isto foi observado mesmo quando as instruções geraram variação comportamental antes das mudanças nas contingências de reforço (Condição MI) e mesmo quando o comportamento não-verbal estabelecido por instruções deixou de ser reforçado na Fase 2.

Estes resultados, mostrando que a história de variação comportamental gerada por diferentes instruções não produziu desempenho sensível à mudança nas contingências de reforço na Condição MI, são inconsistentes com os obtidos por LeFrancois e colaboradores (1988). De acordo com Paracampo e colaboradores (2001), as diferenças entre os procedimentos usados nesses estudos podem ter contribuído para as diferenças de resultados no que concerne à sensibilidade do comportamento instruído à mudança nas contingências de reforço.

Por exemplo, no estudo de Paracampo e colaboradores (2001), a mudança da Fase de Treino, (Fase 1, na qual a história de variação foi construída) para a Fase de Teste (Fase 2, na qual a sensibilidade do comportamento instruído à mudança nas contingências foi avaliada) não foi sinalizada, enquanto no estudo de LeFrancois e colaboradores (1988) foi sinalizada pela apresentação de uma instrução mínima que especificava que os participantes deveriam descobrir qual a melhor maneira de ganhar pontos.

Assim, é possível que o desempenho sensível às contingências de reforço observado na Fase de Teste do estudo de LeFrancois e colaboradores (1988) tenha ocorrido não apenas devido à história de variação comportamental, mas devido a uma interação entre os efeitos dessa história de variação e os efeitos das instruções apresentadas imediatamente antes do início da Fase de Teste.

Esta análise pressupõe que as crianças da Condição Múltiplas Instruções do estudo de Paracampo e colaboradores (2001), possivelmente, também teriam mostrado um desempenho sensível à mudança nas contingências de reforço, caso a mudança nessas contingências também tivesse sido sinalizada por uma instrução mínima que especificasse que os participantes deveriam descobrir qual a melhor maneira de ganhar fichas.

O presente estudo pretendeu avaliar esta possibilidade. Ou seja, o presente estudo objetivou fazer uma replicação sistemática das Condições Instrução e Múltiplas Instruções do estudo de Paracampo e colaboradores (2001) a fim de verificar se os comportamentos não-verbal e verbal de crianças mudam ou não acompanhando a mudança nas contingências de reforço programadas para o comportamento não-verbal, quando: 1) em uma condição, o comportamento não-verbal era estabelecido por instruções correspondentes às contingências de reforço e na outra, o comportamento não-verbal era exposto à variabilidade tanto nas instruções quanto nas contingências; e, 2) nas duas condições, a mudança nas contingências era sinalizada pela apresentação de uma instrução mínima especificando que o participante deveria descobrir qual a melhor maneira de ganhar fichas.

\section{Método}

\section{Participantes}

Participaram do estudo 14 crianças de ambos os sexos $(6$ meninos e 8 meninas), com idades variando entre 8 e 9 anos, cursando a $2^{\mathrm{a}}$ série do ensino fundamental em uma escola pública federal. De cada turma eram escolhidas, pela professora, no máximo 2 crianças. As crianças de uma mesma turma sempre eram atribuídas a condições experimentais diferentes. A participação de todas as crianças foi previamente autorizada pelos responsáveis através de um formulário de consentimento assinado pelos mesmos.

\section{Material}

Foi utilizada uma mesa retangular cujo tampo tinha uma abertura de 49,5 x 49,5 cm. Encaixada sobre esta abertura, estava uma placa em acrílico leitoso e sob esta, 6 lâmpadas fluorescentes de 20 watts, 2 de cor vermelha, 2 de cor verde e 2 de cor amarela. Separando o experimentador do participante, sobre a mesa, havia um anteparo de madeira de $14 \mathrm{~cm}$ de altura e $69,5 \mathrm{~cm}$ de comprimento. Atrás do anteparo, do lado do experimentador, ficavam os arranjos de estímulos previamente preparadas, e um conjunto de interruptores para 
o controle das lâmpadas fluorescentes. $\mathrm{Na}$ frente do anteparo, próximo ao participante, ficava 1 gravador de fita.

Foram utilizados como estímulos discriminativos e condicionais 45 desenhos coloridos de objetos conhecidos das crianças (Ex.: 1 bola, 1 lua, 1 meia etc.). Estes desenhos de $5 \times 5 \mathrm{~cm}$ cada eram impressos em cartões de cartolina que eram colados em folhas de papel cartão de 14 x $14 \mathrm{~cm}$, de maneira a formar 30 diferentes arranjos de estímulos. Cada arranjo de estímulo continha 3 cartões com desenhos; 2 desenhos eram sempre iguais entre si e o terceiro era diferente. Um cartão contendo 1 dos desenhos iguais era colado no topo da folha (estímulo modelo) e os outros 2 mais abaixo e lado a lado (estímulos de comparação). A combinação dos estímulos era aleatória, assim como a ordem de apresentação dos 30 arranjos. Como estímulos contextuais foram utilizadas lâmpadas coloridas acesas, e como estímulos reforçadores, fichas pretas que poderiam ser trocadas por brinquedos e guloseimas. Foram utilizados 2 copos plásticos descartáveis para guardar as fichas. Os copos ficavam sobre a mesa; 1, ao lado esquerdo do participante, e o outro, ao lado direito do experimentador.

O desempenho dos participantes era registrado por um observador em um protocolo de registro previamente preparado e era também gravado em vídeo e em fitas cassete, para análises posteriores.

\section{Situação Experimental}

O experimento foi realizado em uma sala da escola, medindo $48 \mathrm{~m}^{2}$. A sala estava equipada com um condicionador de ar e no teto estavam instaladas 8 lâmpadas fluorescentes de 40 watts cada uma. $\mathrm{Na}$ sala, além da mesa experimental, havia uma mesa, visível ao participante, sobre a qual ficavam expostos diversos brinquedos e guloseimas. Em cada brinquedo e guloseima estava afixada uma etiqueta de papel com um número impresso (Ex.: 3; 10; 20 etc.), indicando o total de fichas que cada brinquedo e guloseima valia.

O participante era conduzido à sala experimental pelo experimentador, aproximadamente 5 minutos antes do início da sessão, onde lhe eram mostrados os brinquedos e guloseimas, e apresentadas, oralmente, as seguintes instruções iniciais: "Eu te trouxe aqui para nós brincarmos de um jogo. No jogo nós temos esta lojinha com vários brinquedos. Estes brinquedos podem ser comprados com fichas como estas aqui (o experimentador mostrava 5 fichas ao participante). Por exemplo, este carrinho vale 10 fichas, este boneco vale 5 fichas. Durante o jogo você poderá ganhar muitas fichas, e no final do jogo você poderá vir aqui na lojinha e comprar brinquedos com suas fichas. Quanto mais fichas você ganhar, mais brinquedos você poderá comprar. Entendeu?" (esta instrução era repetida mais uma vez). Em seguida, o experimentador dizia: "Agora en vou te mostrar como se compra na lojinha. Eu vou te dar 5 fichas para você fazer uma compra na lojinha. Vamos ver o que você pode comprar com 5 fichas?" Após o participante fazer a compra, o experimentador dizia: "Agora vamospara aquela mesa que en vou te explicar como é o jogo". O participante e o experimentador se dirigiram à mesa experimental, com o participante levando o brinquedo e/ou guloseima que comprou, e era dado início à sessão experimental.

Participante e experimentador ficavam sentados à mesa, frente a frente. Inicialmente, o experimentador apresentava oralmente ao participante uma determinada instrução, em seguida os arranjos de estímulos, e logo após, acendia uma das lâmpadas. As fases experimentais eram realizadas em sucessão, sem intervalo, em uma única sessão, que durava aproximadamente 40 minutos. A transição da Fase 1 para a Fase 2, e da Fase 2 para a Fase 3 era marcada pela mudança nas contingências de reforço e sinalizada pela apresentação de uma instrução mínima especificando que o participante deveria descobrir qual a melhor maneira de ganhar fichas.

Imediatamente após a apresentação de um dos 30 arranjos, e enquanto este ainda estava presente, o experimentador acendia uma das lâmpadas fluorescentes. O participante deveria então responder tocando com o dedo um dos estímulos de comparação. Caso a reposta estivesse de acordo com as contingências de reforço programadas (resposta correta), uma ficha era colocada no copo próximo ao participante, a lâmpada era apagada e o arranjo de estímulos retirado; caso a resposta fosse considerada incorreta, a lâmpada era apagada e o arranjo de estímulos retirado, sem apresentação da ficha. Se o participante não respondesse, passados 5 segundos de sua apresentação, a luz era apagada e o arranjo retirado. Estas seqüências definiam uma tentativa.

Entre uma tentativa e outra havia um intervalo variável de aproximadamente 5 segundos. O número de tentativas era variável a depender da fase experimental e do desempenho do participante. Durante alguns dos intervalos entre tentativas, ao longo da sessão, eram feitas perguntas ao participante sobre o que ele deveria fazer para ganhar fichas, porém suas respostas verbais não eram conseqüenciadas diferencialmente.

\section{Procedimento}

Os participantes foram alocados a duas condições experimentais, representadas na Tabela 1. Foram alocados 6 participantes à Condição Única Instrução e 8 à Condição Múltiplas Instruções. Cada condição era constituída de três fases, sendo que a Fase 1 da Condição Múltiplas Instruções foi subdividida em três passos.

Fase 1 - Estabelecimento das discriminacõos condicionais por instruções correspondentes às contingências de reforço: Esta fase, em qualquer uma das duas condições experimentais, era iniciada com o experimentador apresentando as seguintes orientações preliminares: "O objetivo do jogo é você ganhar muitas fichas para depois comprar brinquedos naquela lö̈nha. Agora, eu vou te explicar 
Tabela 1

Condiçôes Experimentais, Estímulos Contextuais e Respostas não-verbais Reforçadas em cada uma das Fases das duas Condiçôes Experimentais

\begin{tabular}{lcccc}
\hline Condições & Estímulos luzes & \multicolumn{3}{c}{ Respostas reforçadas } \\
Condição única & & Fase 1 & Fase 2 & Fase 3 \\
\cline { 3 - 5 } Instrução & Verde & Igual & Diferente & Igual \\
Cermelha & Diferente & Igual & Diferente \\
\hline \multirow{2}{*}{ Condição } & Verde & Passo 1 & & - \\
& Amarela & Igual & - & - \\
& Diferente & - & - \\
Múltiplas & Passo 2 & - & - \\
& Vermelha & Diferente & - & \\
& Amarela & Igual & - & Igual \\
& Verde & Passo 3 & & Diferente \\
\hline
\end{tabular}

Nota. A palavra "igual" representa a resposta de escolha do estímulo de comparação igual ao modelo. A palavra "diferente" representa a escolha do estímulo de comparação diferente do modelo.

o que você tem que fazer para ganhar fichas". O experimentador apresentava ao participante um arranjo de estímulos. Em seguida, apontava para o cartão modelo e dizia: "Este é o cartão-mãe. Toque com o dedo o cartão-mãe". Depois, apontava para os dois cartões de comparação e dizia: "Estes são os cartões-filho. Toque com o dedo os cartôes-filho". Imediatamente após a apresentação destas orientações preliminares, eram apresentadas a cada participante as instruções específicas correspondentes à condição experimental à qual ele fora designado.

$\mathrm{Na}$ Condição UI (Única Instrução), o experimentador acendia a luz verde e dizia: "Quando a mesa ficar verde você deve tocar com o dedo o filho que éigual à mãe. A mesa está verde, toque o filho que é igual à mãe". Após o participante tocar, o experimentador dizia: "Fazendo isso, voce ganha uma ficha que eu tiro aqui do meu copinho e coloco no seu" (no presente estudo, todas as vezes que esta frase era dita, o experimentador entregava uma ficha ao participante). Depois a luz verde era apagada, a luz vermelha acesa e dito: "Quando a mesa ficar vermelha, você deve tocar com o dedo o filho que é diferente da mäe. A mesa está vermelha, toque o filho que é diferente da mãe". Após o participante tocar, o experimentador dizia: "Fazendo isso, você ganha uma ficha que eu tiro aqui do meu copinho e coloco no seù'. As instruções nesta condição descreviam corretamente as contingências de reforço em vigor. Esta fase era encerrada após a emissão de 10 respostas consecutivas corretas, desde que o participante já tivesse obtido, no mínimo, 30 reforços. A transição da Fase 1 para a Fase 2 era marcada pela mudança nas contingências de reforço e sinalizada pela apresentação das seguintes instruções mínimas: "A partir de agora descubra qual a melhor maneira de ganhar fichas".

$\mathrm{Na}$ Condição MI (Múltiplas Instruções), no Passo 1, o experimentador acendia a luz verde e dizia: "Quando a mesa ficar verde você deve tocar com o dedo o filho que é igual à mãe. A mesa está verde, toque o filho que é igual à mãe". Após o participante tocar, o experimentador dizia: "Fazendo isso, você ganha uma ficha que eu tiro aqui do men copinho e coloco no seu". Depois a luz verde era apagada, a luz amarela acesa e dito: "Quando a mesa ficar amarela. Você deve tocar com o dedo o filho que é diferente da mãe. A mesa está amarela, toque o filho que é diferente da mãe". Após o participante tocar, o experimentador dizia: "Fazendo isso, você ganha uma ficha que eu tiro aqui do meu copinho e coloco no senl'. As instruções apresentadas no início dos Passos 2 e 3 tinham a mesma forma das instruções apresentadas no início do Passo 1, exceto que as cores dos estímulos contextuais variavam. No Passo 2, quando a mesa estivesse amarela o participante deveria tocar o estímulo igual ao modelo e quando a mesa estivesse vermelha o participante deveria tocar o estímulo diferente do modelo; no Passo 3, quando a mesa estivesse verde o participante tocar o estímulo igual ao modelo e quando a mesa estivesse vermelha deveria tocar o estímulo diferente do modelo. As instruções nesta condição descreviam corretamente as contingências de reforço em vigor. Cada passo era encerrado após a apresentação de 20 tentativas. A transição do Passo 3 para a Fase 2 era marcada pela mudança nas contingências de reforço e sinalizada pela apresentação das seguintes instruções mínimas: "A partir de agora descubra qual a melhor maneira de ganhar fichas".

As instruções específicas para cada condição eram apresentadas duas vezes para o participante. Após serem apresentadas, o experimentador informava ao participante que não mais poderia conversar com ele.

Fase 2-Reversão das discriminaçös condicionais: Durante esta fase, para todos os participantes (isto é, qualquer que fosse a condição experimental) eram reforçadas as respostas de apontar para o estímulo de comparação igual ao est́mulo modelo quando a luz vermelha estivesse acesa e as respostas de apontar para o estímulo de comparação diferente do estímulo modelo quando a luz verde estivesse acesa (respostas corretas na Fase 2). Esta fase era encerrada quando um de dois critérios fosse atingido, o que ocorresse primeiro: a) a emissão de 10 respostas consecutivas corretas; ou, b) a ocorrência de 40 tentativas. A transição da Fase 
2 para a Fase 3 também era marcada pela mudança nas contingências de reforço e sinalizada pela apresentação das seguintes instruções mínimas: "A partir de agora descubra qual a melhor maneira de ganhar fichas".

Fase 3 - Retorno às contingências da Fase 1: Durante esta fase eram reforçadas as respostas de apontar para o estímulo de comparação igual ao estímulo modelo quando a luz verde estivesse acesa, e as respostas de apontar para o estímulo de comparação diferente do estímulo modelo quando luz vermelha estivesse acesa (respostas corretas na Fase 3). Esta fase era encerrada quando um de dois critérios fosse atingido, o que ocorresse primeiro: a) a emissão de 10 respostas consecutivas corretas ou, b) a ocorrência de 40 tentativas.

Durante todas as três fases das duas condições, as respostas corretas eram reforçadas em CRF. Respostas incorretas eram conseqüenciadas apenas com a retirada do arranjo de estímulos que havia sido apresentado, seguida pela apresentação de um novo arranjo. Também durante todas as três fases das duas condições, quando uma luz estava acesa, as outras estavam apagadas. Durante a Fase 1 na Condição UI e durante as Fases 2 e 3 nas duas condições, as luzes vermelha e verde eram apresentadas aleatoriamente ao longo das tentativas, garantindose que as duas fossem apresentadas o mesmo número de vezes em cada fase. Durante a Fase 1 na Condição MI, a cada passo as luzes eram apresentadas aleatoriamente no respectivo passo, garantindo-se que cada uma fosse apresentada 10 vezes.

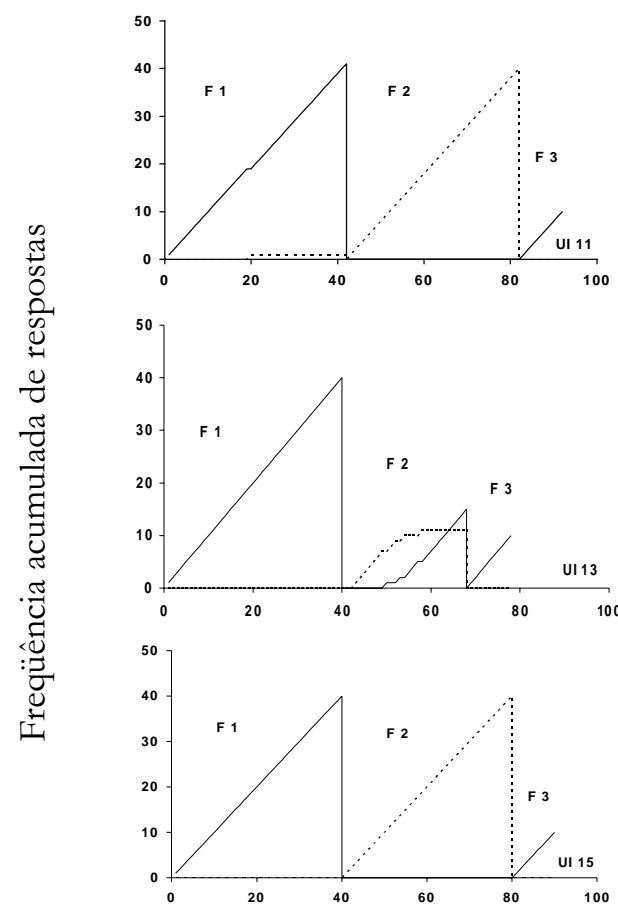

Nas duas condições, perguntas acerca do que o participante deveria fazer para ganhar fichas eram feitas após a $3^{\mathrm{a}}, 7^{\mathrm{a}}, 10^{\mathrm{a}}, 20^{\mathrm{a}}, 40^{\mathrm{a}}$, e/ou a última tentativa de cada fase e/ ou passo. As seguintes perguntas eram apresentadas, uma por vez e em ordem alternada, a cada participante: "O que você deve fazer para ganhar ficha quando a mesa está vermelha?", "O que você deve fazer para ganhar ficha quando a mesa está verde?’ Durante o Passo 1 da Fase 1 da Condição MI a palavra 'vermelha' foi substituída pela palavra 'amarela', durante o Passo 2, a palavra 'verde' foi substituída pela palavra 'amarela'. Caso o participante não respondesse à primeira pergunta dentro de aproximadamente 5 segundos, era feita a segunda pergunta. Após o participante responder à segunda pergunta, ou após os 5 segundos de espera pela sua resposta, o experimentador dizia: "Agora vamos continuar jogando", e iniciava uma nova tentativa. As respostas dos participantes a essas perguntas não eram reforçadas.

\section{Resultados}

\section{Condição Única Instrução}

A Figura 1 mostra o número acumulado de respostas nãoverbais corretas (linha cheia) e incorretas (linha tracejada) apresentadas pelos Participantes UI11, UI12, UI13, UI14, UI15 eUI16 durante as três fases da Condição Única Instrução. Podese observar que todos os 6 participantes expostos a esta condição iniciaram a Fase 1 respondendo corretamente, seguindo a
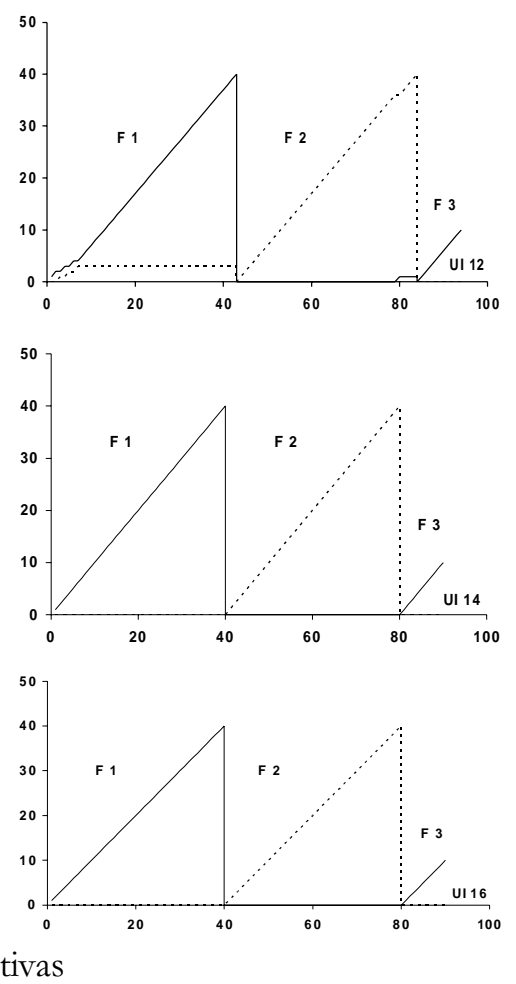

Figura 1. Freqüência acumulada de respostas não-verbais corretas (linha sólida) e incorretas (inha tracejada), para cada participante (UI) da Condição 'Única Instrução', durante cada fase (F) experimental. Quebras na curva acumulada indicam mudanças de fase. $\mathrm{Na}$ Fase 1 , as instruções correspondiam às contingências. As contingências em vigor na Fase 1 eram revertidas na Fase 2, tornando as instruções iniciais discrepantes, e restabelecidas na Fase 3. 
instrução apresentada no início da fase. Ou seja, conforme havia sido descrito na instrução, os participantes escolheram o estímulo de comparação igual ao estímulo modelo na presença da luz verde e o estímulo de comparação diferente do estímulo modelo na presença da luz vermelha. O Participante UI12 não seguiu a instrução na $3^{a}, 5^{a}$ e $7^{a}$ tentativas desta fase.

Na Fase 2, 5 (UI11, UI12, UI14, UI15 e UI16) dos 6 participantes continuaram seguindo instruções, independentemente da mudança nas contingências de reforço programadas e desta mudança ter sido sinalizada. Portanto, durante a Fase 2, suas respostas não-verbais tornaram-se incorretas e a fase foi encerrada pelo número máximo (40) de tentativas. O Participante UI13 iniciou a Fase 2 seguindo a instrução apresentada no início da Fase 1 . A partir da $10^{a}$ tentativa o seu desempenho não-verbal começou a variar e continuou variando até a $19^{a}$ tentativa, quando então passou a ficar sob controle das contingências de reforço programadas na Fase 2. Ou seja, este participante passou a escolher o estímulo de comparação diferente do modelo na presença da luz verde e o estímulo de comparação igual ao modelo na presença da luz vermelha, que era o desempenho correto, que produzia fichas nesta fase.

Na Fase 3, os Participantes UI11, UI12, UI14, UI15 e UI16 continuaram apresentando o mesmo desempenho não-verbal apresentado nas fases anteriores. Como as contingências de reforço programadas para a Fase 3 eram as mesmas programadas para a Fase 1, as respostas não-verbais dos participantes voltaram a ficar de acordo com as contingências, tornando-se corretas. $\mathrm{O}$ Participante UI13, novamente mudou seu desempenho nãoverbal, passando a responder de acordo com as contingências de reforço programadas na Fase 3. Ou seja, passou a escolher o estímulo de comparação igual ao modelo na presença da luz verde e o estímulo de comparação diferente do modelo na presença da luz vermelha.

No presente estudo, as respostas dos participantes às perguntas do experimentador foram categorizadas como verbalizações corretas e incorretas. As verbalizações corretas foram definidas como descrições da resposta de escolha segundo o modelo que produzia reforço quando emitida na presença do estímulo contextual especificado na pergunta. As verbalizações incorretas foram definidas como descrições da resposta de escolha segundo o modelo que não produzia reforço quando emitida na presença do estímulo contextual especificado na pergunta.

Todos os participantes da Condição UI descreveram corretamente as respostas de escolha que produziam fichas na Fase 1. Na Fase 2, os Participantes UI11, UI12, UI14, UI15 e U116 apresentaram respostas verbais incorretas, uma vez que continuaram descrevendo as respostas não-verbais que produziam fichas na Fase 1. O Participante UI13, na Fase 2, apresentou verbalizações incorretas após a apresentação da $3^{\mathrm{a}}$ e da $7^{\mathrm{a}}$ tentativa, quando foram feitas a primeira e a segunda apresentação do par de perguntas, respectivamente. Mas apresentou verbalizações corretas, isto é, descreveu as respostas de escolha que produziam fichas nesta Fase 2, após a apresentação da $10^{\mathrm{a}}$ e da $20^{\mathrm{a}}$ tentativa, quando foram feitas a $3^{\mathrm{a}}$ e a última apresentação do par de perguntas, respectivamente. $\mathrm{Na}$ Fase 3 , todos os participantes apresentaram verbalizações corretas todas as vezes que foram solicitados a responder às perguntas.

O comportamento verbal, de todos os participantes, correspondeu ao não-verbal durante as três fases desta condição, independente de se o comportamento não-verbal estava ou não de acordo com as contingências de reforço programadas. A correspondência entre o comportamento verbal e o não-verbal dos Participantes UI11, UI12, UI14, UI15 e UI16 observada na Fase 1, permaneceu inalterada durante a Fase 2, quando a contingência foi mudada. Ou seja, na Fase 2, tanto o comportamento verbal quanto o não-verbal destes 5 participantes não mudaram acompanhando a mudança nas contingências de reforço programadas. Na Fase 3, todos os 5 participantes continuaram apresentando os mesmos comportamentos, verbal e não-verbal, apresentados nas Fases 1 e 2 . O comportamento verbal do Participante UI13, correspondeu ao não-verbal durante a Fase 1 e mudou acompanhando as mudanças nas contingências de reforço programadas nas Fases 2 e 3.

\section{Condição Múltiplas Instruções}

A Fase 2 desta condição estava programada para ser encerrada quando um dos seguintes critérios fosse atingido, o que ocorresse primeiro: a) a emissão de 10 respostas consecutivas corretas; ou b) a ocorrência de 40 tentativas. Isto ocorreu para 7 (MI21, MI22, MI23, MI24, MI25, MI27 e MI28) dos 8 participantes. No entanto, na Fase 2, o Participante MI26 foi exposto a 63 tentativas, porque a partir da $35^{2}$ tentativa desta fase, quando respondeu corretamente pela segunda vez, ele variou um pouco seu desempenho, apresentando ora respostas corretas, ora respostas incorretas. Dessa forma, na Fase 2 este participante foi exposto a um número maior de tentativas que os demais para verificar se o seu desempenho se estabilizaria em um padrão de respostas corretas ou em um padrão de respostas incorretas. Para que o comportamento verbal do Participante MI26 pudesse ser registrado durante essas tentativas adicionais a que foi exposto, além das 5 apresentações programadas do par de perguntas, foram feitas mais 2 apresentações do par de perguntas, uma após a $60^{a}$ tentativa e a outra a última tentativa.

A Condição Múltiplas Instruções gerou, nas três fases, os dados mostrados na Figura 2. Observa-se nesta figura que sete (MI21,MI22,MI23,MI24,MI26,MI27 eMI28) dos 8 participantes desta condição seguiram as instruções apresentadas no início dos Passos 1, 2 e 3. Contudo, o Participante MI28 emitiu duas respostas incorretas no Passo 1 , na $2^{\mathrm{a}}$ e $10^{\mathrm{a}}$ tentativas. $\mathrm{O}$ Participante MI25 seguiu as instruções no Passo 1. Entretanto, não iniciou o Passo 2 seguindo as instruções. Iniciou o Passo 2 emitindo respostas incorretas nas 2 primeiras tentativas. A partir da $3^{\text {a }}$ tentativa passou a apresentar um desempenho variável, ora 

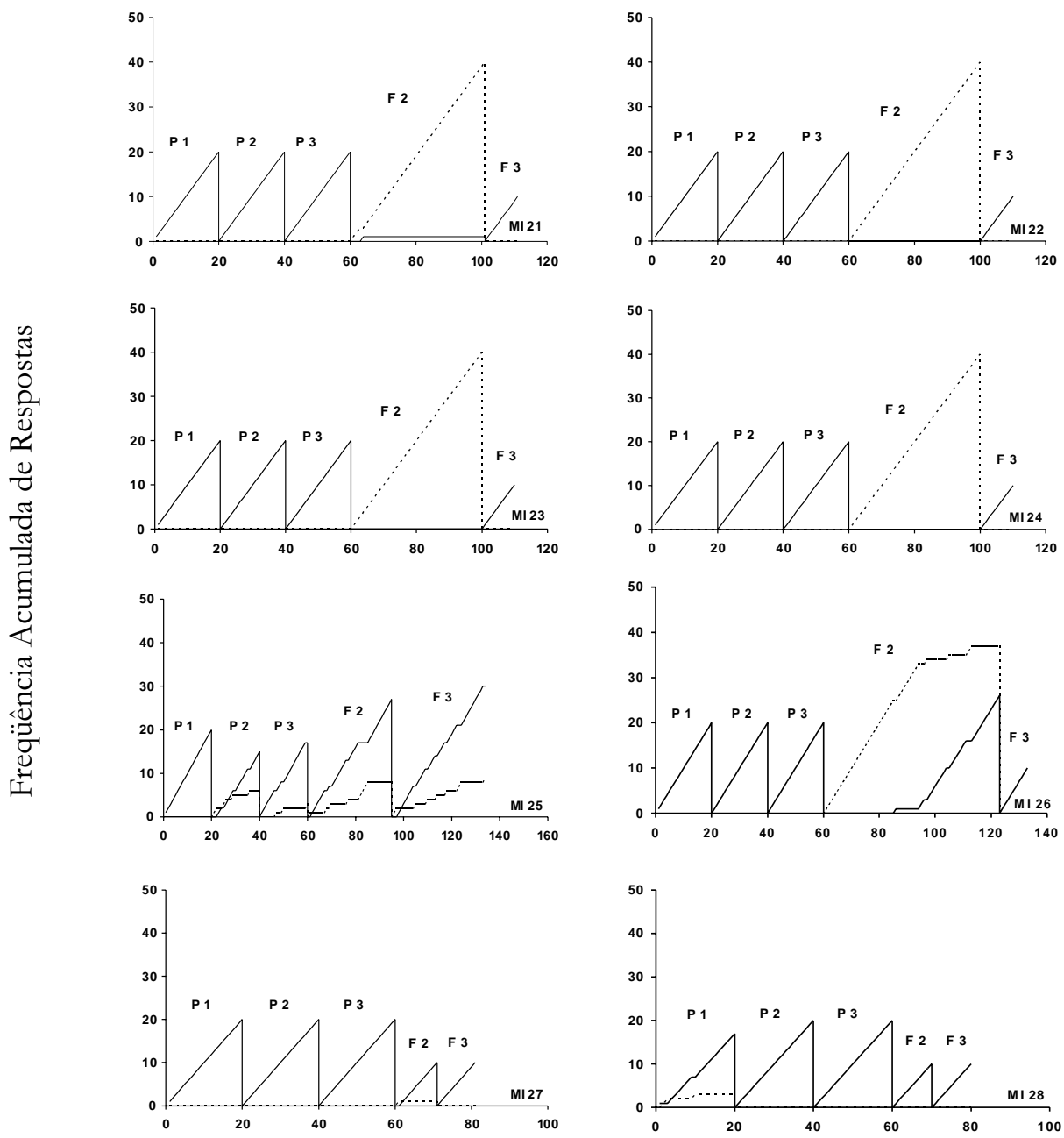

Tentativas

Figura 2. Freqüência acumulada de respostas não verbais corretas (linha sólida) e incorretas (linha tracejada), para cada participante (MI) da Condição 'Múltiplas Instruções', durante cada passo (P) da Fase 1 e nas Fases 2 e 3. Quebras na curva acumulada indicam mudanças de passo e de fase (F). Na Fase 1, as instruções dos Passos 1, 2 e 3 correspondiam às contingências e variavam entre os passos. As contingências em vigor no Passo 3 eram revertidas na Fase 2, tornando as instruções do Passo 3 discrepantes, e restabelecidas na Fase 3.

emitindo respostas corretas, ora emitindo respostas incorretas. No Passo 3, seguiu as instruções apresentadas no início do passo, emitindo três respostas incorretas durante o passo, na $7^{\mathrm{a}}, 10^{\mathrm{a}}$ e $20^{\mathrm{a}}$ tentativas.

$\mathrm{Na}$ Fase 2, quando as contingências foram mudadas e essa mudança foi sinalizada, o comportamento não-verbal de 4 participantes (MI21, MI22, MI23 eMI24) permaneceu inalterado. Isto é, o comportamento não-verbal dos Participantes MI21, MI22, MI23 e MI24 não mudou acompanhando a mudança nas contingências. Estes participantes continuaram emitindo as mesmas respostas não-verbais que haviam emitido no Passo 3. Assim, seus desempenhos tornaram-se incorretos. Em contraste, os Participantes MI25, MI26, MI27 e MI28 mudaram seus desempenhos não-verbais acompanhando a mudança nas contingências e passaram a responder corretamente, de acordo com as contingências em vigor na Fase 2 . Os Participantes MI25 e MI26 iniciaram a Fase 2 seguindo as instruções apresentadas no início do Passo 3. Depois, o Participante MI25 passou a responder corretamente nas 6 tentativas que se seguiram àinicial. $\mathrm{Da} 8^{\mathrm{a}}$ a $25^{\mathrm{a}}$ tentativa variou seu desempenho, apresentando ora respostas corretas (nas tentativas 9, 11, 12, 13, 14, 15, 16, 18, 19, 20 e 21), ora respostas incorretas (nas tentativas $8,10,17,22$, 23,24 e 25). Da $26^{a}$ tentativa em diante, passou a responder corretamente, de acordo com as contingências de reforço programadas na Fase 2, e atingiu o critério de 10 respostas consecutivas corretas para o encerramento desta fase. Já o Participante MI26 continuou seguindo as instruções até a $25^{\mathrm{a}}$ tentativa. $\mathrm{Na} 26^{\mathrm{a}}$ tentativa respondeu corretamente, mas voltou 
a seguir a instrução da $27^{a}$ a $34^{a}$ tentativa. Da $35^{a}$ a $53^{a}$ tentativa variou o seu desempenho, apresentando ora respostas corretas (nas tentativas 35, 36, 38, 39, 40, 41, 42, 43, 44, 46, 47, 48, 49, $50 \mathrm{e}$ 51), ora respostas incorretas (nas tentativas 37, 45, 52 e 53). Da $54^{a}$ tentativa em diante, passou a responder corretamente, de acordo com as contingências de reforço programadas na Fase 2. Os Participantes MI27 e MI28 apresentaram comportamentos não-verbais de acordo com as contingências em vigor na Fase 2 logo no início desta fase, a partir da $2^{\mathrm{a}} \mathrm{e}$ da $1^{\text {a }}$ tentativa, respectivamente.

Na Fase 3, os Participantes MI21, MI22, MI23 e MI24 continuaram apresentando o mesmo comportamento não-verbal apresentado no Passo 3 e na Fase 2. Os Participantes MI26, MI27 e MI28 mais uma vez mudaram seus desempenhos de acordo com a mudança nas contingências na Fase 3. Como as contingências de reforço programadas para a Fase 3 eram as mesmas programadas para o Passo 3, os desempenhos destes 7 participantes (MI21, MI22, MI23, MI24, MI26, MI27 e MI28) tornaram-se corretos. Tal como os Participantes MI26, MI27 e MI28, o Participante MI25 também mudou seu desempenho de acordo com a mudança nas contingências na Fase 3, mas apresentou um desempenho mais variável do que os Participantes MI26, MI27 e MI28. Isto é, o Participante MI25 respondeu incorretamente em 9 (nas tentativas 1, 2, 10, 15, 19, 23, 28, 29 e 40) das 40 tentativas a que foi exposto nesta fase. Portanto, respondeu corretamente, de acordo com as contingências de reforço programadas na Fase 3, na maior parte das tentativas desta fase.

Todos os participantes da Condição MI apresentaram verbalizações corretas todas as vezes que o par de perguntas foi feito em cada um dos passos da Fase 1. Ou seja, descreveram corretamente as respostas não-verbais que produziam fichas nos Passos 1, 2 e 3.

Na Fase 2, os Participantes MI21, MI22, MI23 e MI24 apresentaram respostas verbais incorretas, uma vez que continuaram descrevendo as respostas não-verbais que haviam produzido fichas durante o Passo 3. O Participante MI25, após a apresentação da $3^{a}$ tentativa, quando foi feita a primeira apresentação do par de perguntas nesta fase, descreveu incorretamente as respostas não-verbais que produziam fichas durante a Fase 2 . A partir da $7^{a}$ tentativa, isto é, da segunda apresentação do par de perguntas em diante, passou a apresentar verbalizações corretas quando as perguntas foram feitas. Ou seja, passou a descrever as respostas não-verbais que produziam fichas na Fase 2. $\mathrm{O}$ Participante MI26 apresentou um desempenho similar, isto é, apresentou verbalizações incorretas após a $3^{\mathrm{a}}, 7^{\mathrm{a}}, 10^{\mathrm{a}}$ e $20^{\mathrm{a}}$ tentativas (quando foram feitas a $1^{a}, 2^{a}, 3^{a}$ e $4^{a}$ apresentação do par de perguntas, respectivamente), e apresentou verbalizações corretas após a 40a, $60^{\mathrm{a}}$ e $63^{\mathrm{a}}$ tentativas (quando foram feitas a $5^{a}, 6^{a}$ e a última apresentação do par de perguntas, respectivamente). Os Participantes MI27 e MI28 apresentaram verbalizações corretas desde a primeira apresentação do par de perguntas. Ou seja, descreveram as respostas não-verbais que produziam fichas na Fase 2.

Na Fase 3, os Participantes MI21, MI22, MI23 e MI24 continuaram apresentando as mesmas respostas verbais que haviam apresentado em resposta às perguntas do experimentador no Passo 3 e na Fase 2. Como as contingências de reforço programadas para a Fase 3 eram as mesmas programadas para $O$ Passo 3 , as respostas verbais destes participantes voltaram a ficar de acordo com as contingências, tornando-se corretas. Os Participantes MI25, MI26, MI27 e MI28, com a mudança nas contingências na Fase 3, passaram a descrever corretamente as respostas não-verbais que produziam fichas na Fase 3, desde a primeira apresentação do par de perguntas. Portanto, nesta fase, os Participantes MI25, MI26, MI27 e MI28 apresentaram verbalizações corretas todas as vezes que as perguntas foram feitas.

O comportamento verbal dos Participantes MI21, MI22, MI23 e MI24 correspondeu ao não-verbal durante as três fases desta condição, independente de se o comportamento não-verbal estava ou não de acordo com as contingências de reforço programadas. A correspondência entre o comportamento verbal e o nãoverbal dos Participantes MI21, MI22, MI23 e MI24 observada no Passo 3, permaneceu inalterada durante a Fase 2, quando a contingência foi mudada. Ou seja, na Fase 2, tanto o comportamento verbal quanto o não-verbal destes 4 participantes não mudaram acompanhando a mudança nas contingências de reforço programadas. Na Fase 3, todos estes 4 participantes continuaram apresentando os mesmos comportamentos, verbal e não-verbal, apresentados no Passo 3 e na Fase 2.

Comparando as respostas verbais e não-verbais dos Participantes MI26, MI27 e MI28 observa-se que o comportamento verbal destes participantes correspondeu ao não-verbal durante as três fases desta condição e todos estes 3 participantes mudaram seus desempenhos acompanhando as mudanças nas contingências de reforço programadas nas Fases 2 e 3. Tal como os Participantes MI26, MI27 e MI28, o Participante MI25 também apresentou comportamento verbal correspondente ao não-verbal durante os Passos 1, 2 e 3, e tanto o seu comportamento verbal quanto o não-verbal também mudaram acompanhando as mudanças na contingências de reforço programadas nas Fases 2 e 3. Mas diferente dos Participantes MI26, MI27 e MI28, nas Fases 2 e 3, o comportamento verbal do Participante MI25 nem sempre correspondeu ao não-verbal. Ou seja, o Participante MI25, na Fase 2, apresentou respostas não-verbais corretas na $2^{\mathrm{a}} \mathrm{e} 3^{\mathrm{a}}$ tentativas e, após a $3^{\mathrm{a}}$ tentativa, quando foi feita a primeira apresentação do par de perguntas, apresentou verbalizações incorretas. Depois, continuou apresentando respostas não-verbais corretas até a $7^{a}$ tentativa e, após a $7^{\mathrm{a}}$ tentativa, quando foi feita a segunda apresentação 
do par de perguntas, apresentou verbalizações corretas e correspondentes ao seu comportamento não-verbal. Após a $10^{\mathrm{a}}$ tentativa, quando foi feita a terceira apresentação do par de perguntas, continuou apresentando verbalizações corretas, mas apresentou respostas não-verbais incorretas na $8^{\mathrm{a}} \mathrm{e} 10^{\mathrm{a}}$ tentativas. Finalmente, após a $20^{a}$ tentativa, quando foi feita a quarta e última apresentação do par de perguntas, apresentou verbalizações corretas e correspondentes ao seu comportamento não-verbal. $\mathrm{Na}$ Fase 3, verbalizou corretamente, descrevendo as respostas não-verbais que produziam fichas na Fase 3 , todas as vezes que as perguntas foram feitas nesta fase (após a $3^{\mathrm{a}}, 7^{\mathrm{a}}, 10^{\mathrm{a}}, 20^{\mathrm{a}}$ e $40^{\mathrm{a}}$ tentativas) e apresentou respostas não-verbais incorretas em 9 tentativas (tentativas $1 ; 2 ; 10 ; 15 ; 19 ; 23 ; 28 ; 29$ e 40). Deste modo, o seu comportamento verbal ora correspondeu ora não correspondeu ao comportamento não-verbal.

Em síntese, os resultados mostraram que tanto o comportamento não-verbal quanto o verbal de 5 (UI11, UI12, UI14, UI15 e UI16) dos 6 participantes da Condição Única Instrução (UI) e de 4 (MI21, MI22, MI23 e MI24) dos 8 participantes da Condição Múltiplas Instruções (MI) não mudaram acompanhando a mudança nas contingências de reforço programadas. Portanto, 1 (UI13) dos 6 participantes da Condição Ule 4 (MI25,MI26,MI27 e MI28) dos 8 participantes da Condição MI, mudaram tanto o comportamento não-verbal quanto o verbal acompanhando a mudança nas contingências de reforço programadas no experimento.

\section{Discussão}

Os resultados da Condição Única Instrução (UI) do presente estudo, mostrando que tanto o comportamento nãoverbal quanto o verbal de 5 (UI11, UI12, UI14, UI15 e UI16) dos 6 participantes dessa condição não mudaram acompanhando a mudança nas contingências são similares aos resultados da Condição Instrução do estudo de Paracampo e colaboradores (2001) e aos das Condições 3 e 4 do estudo de LeFrancois e colaboradores (1988). Juntos estes resultados indicam que a sinalização da mudança nas contingências de reforço programadas, pela apresentação de uma instrução mínima que especifica que se deve descobrir qual a melhor maneira de ganhar fichas, não é uma condição, por si só, suficiente para gerar um desempenho instruído sensível à mudança nas contingências. Isto considerando que a mudança nas contingências de reforço programadas foi sinalizada no presente estudo e nas Condições 3 e 4 do estudo de LeFrancois e colaboradores, mas não foi sinalizada no estudo de Paracampo e colaboradores.

Já os resultados da Condição Múltiplas Instruções (MI) do presente estudo diferem dos resultados obtidos na Condição MI do estudo de Paracampo e colaboradores (2001) e dos resultados obtidos nas Condições 1 e 2 do estudo de LeFrancois e colaboradores (1988), quanto ao percentual de participantes que apresentaram desempenho sensível à mudança nas contingências. Na Condição MI do presente estudo, 4 (MI25, MI26, MI27 e MI28) dos 8 participantes (i.e., $50 \%$ dos participantes) apresentaram um desempenho sensível à mudança nas contingências, enquanto que 1 dos 6 participantes (i.e., $16.6 \%$ ) da Condição MI do estudo de Paracampo e colaboradores e 25 dos 30 participantes (i.e., $83.3 \%$ ) das Condições 1 e 2 do estudo de LeFrancois e colaboradores, fizeram o mesmo. Assim, quando se comparam os resultados da Condição MI do presente estudo com os resultados da Condição MI do estudo de Paracampo e colaboradores, pode-se sugerir que a interação entre os efeitos dessa história de variação e os efeitos da sinalização da mudança nas contingências, contribuiu para que os Participantes MI25, MI26, MI27 e MI28 da Condição MI do presente estudo tivessem apresentado um desempenho sensível à mudança nas contingências. Entretanto, não fica claro porque 4 (MI21, MI22, MI23 e MI24) dos 8 participantes da Condição MI do presente estudo apresentaram um desempenho insensível à mudança nas contingências. Diferenças entre os desempenhos de participantes expostos a uma mesma condição experimental, principalmente quando os seus desempenhos mantêm contato com a discrepância regra / conseqüências programadas, também têm sido encontradas em outros estudos (Catania \& cols., 1982; Cerutti, 1991, 1994; Galizio, 1979; Hayes \& cols., 1986a, 1986b; Joyce \& Chase, 1990; LeFrancois e colaboradores, 1988; Lowe, 1979; Michael \& Bernstein, 1991; Paracampo \& cols., 1993; Shimoff \& cols., 1981; Torgrud \& Holborn, 1990) e não tem sido suficientemente esclarecidas na literatura. A variabilidade sugere controle experimental fraco pelas variáveis manipuladas pelo experimentador, que possivelmente compete com outras variáveis não planejadas (Sidman, 1960). Assim, é possível que as diferenças entre os desempenhos dos participantes da Condição MI tenham ocorrido devido a diferenças em suas histórias pré-experimentais, mas também há a possibilidade de que possíveis diferenças nas fontes de controle dos desempenhos dos participantes na Fase 1 tenham contribuído para a ocorrência daquelas diferenças.

Em outras palavras, quando se analisam apenas os dados da Fase 1 da Condição MI, pode-se dizer que todos os 8 participantes desta condição responderam aos estímulos de comparação nos Passos 1, 2 e 3 sob controle de regras, uma vez que mudaram seus desempenhos de acordo com as mudanças das regras. Contudo, quando também se analisam os dados da Fase 2, mostrando que os Participantes MI21, MI22, MI23 e MI24 apresentaram um desempenho insensível, enquanto que os Participantes MI25, MI26, MI27 e MI28 apresentaram um desempenho sensível à mudança nas contingências, pode-se sugerir que possivelmente os Participantes MI21, MI22, MI23 e MI24 responderam nos Passos 1, 2 e 3 inteiramente sob controle de regras; enquanto 
que os Participantes MI25, MI26, MI27 e MI28 responderam nos Passos 1, 2 e 3 sob controle da interação entre regras e conseqüências programadas. Ou seja, é possível que os Participantes MI21, MI22, MI23 e MI24 tenham seguido regra nos Passos 1,2 e 3, independentemente de suas respostas serem ou não reforçadas; enquanto que os Participantes MI25, MI26, MI27 eMI28 tenham seguido regra nos Passos 1,2e3 dependendo de suas respostas produzirem ou não reforço. Admitindo essa possibilidade, pode-se sugerir que os Participantes MI21, MI22, MI23 e MI24 apresentaram um desempenho na Fase 2 sob controle da regra, previamente apresentada no Passo 3. Diferente dos Participantes MI25, MI26, MI27 e MI28, que apresentaram um desempenho na Fase 2 sob controle das conseqüências programadas nesta fase, possivelmente devido às suas histórias de exposição às mudanças nas regras e nas contingências de reforço programadas e da sinalização da mudança nas contingências quando mantiveram contato com a discrepância regra / conseqüências programadas.

Considerando esta análise, é possível que os Participantes MI21, MI22, MI23 e MI24 tenham aprendido nos Passos 1, 2 e 3 as discriminações condicionais específicas, estabelecidas por regras em cada um desses passos, isto é, tenham aprendido a escolher o igual na presença da luz verde ou da amarela e a escolher o diferente na presença da luz vermelha ou da amarela, dependendo da regra apresentada no início do passo e independentemente de suas respostas serem ou não reforçadas; enquanto que os Participantes MI25, MI26, MI27 e MI28 tenham aprendido a escolher o igual ou o diferente na presença de uma luz e tenham aprendido também que suas respostas produziam ou não reforço, e que isso ocorria qualquer que fosse a cor do estímulo contextual, se vermelha, verde ou amarela. Assim, quando o seguimento de regra deixou de ser reforçado na Fase 2 e essa mudança nas contingências foi sinalizada pela apresentação de uma instrução mínima que especificava que se deveria descobrir qual a melhor maneira de ganhar fichas, os Participantes MI21, MI22, MI23 eMI24 permaneceram seguindo regra, independentemente da mudança nas contingências e desta mudança ter sido sinalizada, e os Participantes MI25, MI26, MI27 e MI28 mudaram os seus desempenhos e passaram a responder de acordo com as novas contingências de reforço.

Quanto ao comportamento verbal, Paracampo e colaboradores (2001) sugerem que a observação da correspondência entre comportamento verbal e não-verbal antes da mudança nas contingências de reforço programadas não é suficiente para se afirmar que o verbal controla o não-verbal. Para tanto, seria necessário observar a manutenção dessa correspondência, na ausência de reforço, após a mudança nas contingências. E mesmo assim, ainda seria necessário avaliar a possibilidade dessa correspondência estar sob controle de uma terceira variável, como por exemplo, de regras. De acordo com esta proposição, pode-se sugerir que tanto o comportamento verbal quanto o não-verbal dos Participantes UI11, UI12, UI14,
UI15 e UI16, expostos a Condição UI, e dos Participantes MI21, MI22, MI23 e MI24, expostos a Condição MI, estavam sob controle das regras, previamente apresentadas no início da Fase 1 (caso dos participantes da Condição UI) e no início do Passo 3 (caso dos participantes da Condição MI). Isto considerando que, apesar da correspondência entre o comportamento não-verbal e o verbal, observada na Fase 1 (caso dos participantes da Condição UI) e no Passo 3 (caso dos participantes da Condição MI), ter se mantido inalterada na Fase 2, ou seja, apesar desta correspondência ter sido mantida na ausência de reforço, o comportamento não-verbal destes participantes foi estabelecido previamente por regras e, portanto, não se pode descartar a possibilidade de que tanto o comportamento verbal quanto o não-verbal estivessem sob controle das regras, previamente apresentadas no início da Fase 1 (participantes da Condição UI) e no início do Passo 3 (participantes da Condição MI).

Por outro lado, analisando as interações entre comportamento verbal e comportamento não-verbal dos Participantes UI13, MI25, MI26, MI27 e MI28 pode-se sugerir que tanto o comportamento verbal quanto o não-verbal destes participantes estavam sob controle das conseqüências programadas na Fase 2, uma vez que tanto o comportamento não-verbal quanto o comportamento verbal que descrevia o não-verbal, mudaram quando as contingências foram alteradas.

Em síntese, esta análise sugere que uma história de variação comportamental, gerada pela apresentação de diferentes instruções, pode interferir na sensibilidade do comportamento de seguir regras à mudança nas contingências de reforço. Mas isto tende mais a ocorrer quando a mudança nas contingências é sinalizada do que quando não é sinalizada pela apresentação de uma instrução mínima, especificando que o participante deve descobrir qual a melhor maneira do seu comportamento ser reforçado. Não fica claro, contudo, que características uma história de variação comportamental deveria apresentar, em relação às condições de teste, para que os seus efeitos pudessem ser observados em um maior número de participantes. Neste sentido, a função da história de variação comportamental, como uma condição que pode facilitar a sensibilidade do seguimento de regras às contingências de reforço programadas, deveria continuar sendo investigada.

\section{Referências}

Albuquerque, L. C. (2001). Definições de regras. Em H. J. Guilhardi, M. B. B. P. Madi, P. P. Queiroz \& M. C. Scoz (Orgs.), Sobre comportamento e cognição: Expondo a variabilidade (pp.132-140). Santo André, SP: ARBytes.

Albuquerque, L. C. (2002). Condições sob as quais pode-se dizer que um comportamento é controlado por regras [Resumos]. Em Associação Brasileira de Psicoterapia e Medicina Comportamental (Org.), Anais, XI Encontro Brasileiro de Psicoterapia e Medicina Comportamental (pp. 275-276). Londrina, PR: ABPMC.

Albuquerque, L. C., de Souza, D. G., Matos, M. A. \& Paracampo, C. C. P. (2003). Análise dos efeitos de histórias experimentais sobre o seguimento subseqüente de regras. Acta Comportamentalia, 11, 87-126. 
Albuquerque, L. C. \& Ferreira, K. V. D. (2001). Efeitos de regras com diferentes extensões sobre o comportamento humano. Psicologia: Reflexão e Crítica, 14, 143-155.

Albuquerque, L. C., Matos, M. A, de Souza, D. G. \& Paracampo, C. C. P. (no prelo). Investigação do controle por regras e do controle por histórias de reforço sobre o comportamento humano. Psicologia: Reflexão e Crítica.

Baron, A., Kaufman, R. \& Stauber. K. A. (1969). Effects of instructions and reinforcement-feedback on human operant behavior maintained by fixedinterval reinforcement. Journal of the Experimental Analysis of Behavior, 12, 701 712.

Catania, A. C. (1999). Aprendizagem: Comportamento, linguagem e cognição (D. G. de Souza, Trad.). Porto Alegre: Edições 4/ Artes Médicas. (Original publicado em 1998)

Catania, A. C., Matthews, A. \& Shimoff, E. (1982). Instructed versus shaped human verbal behavior: Interactions with nonverbal responding. Journal of the Experimental Analysis of Behavior, 38, 233-248.

Catania, A. C., Matthews, A. \& Shimoff, E. (1990). Properties of rule-governed behaviour and their implications. Em D. E. Blackman \& H. Lejeune (Orgs.), Behaviour analysis in theory and practice: Contributions and controversies (pp.215230). Brighton: Lawrence Erlbaum.

Cerutti, D. T. (1991). Discriminative versus reinforcing properties of schedules as determinants of schedule insensitivity in humans. The Psychological Record, 41, 51-67.

Cerutti, D. T. (1994). Compliance with instructions: Effects of randomness in scheduling and monitoring. The Psychological Record, 44, 259-269.

Chase, P. N. \& Danforth, J. S. (1991). The role of rules in concept learning. Em L. J. Hayes \& P. N. Chase (Orgs.), Dialogues on verbal behavior (pp. 205-225). Hillsdale, NJ: Erlbaum.

Galizio, M. (1979). Contingency-shaped and rule-governed behavior: Instructional control of human loss avoidance. Journal of the Experimental Analysis of Behavior, 31, 53-70.

Hayes, S. C., Brownstein, A. J., Haas, J. R. \& Greenway, D. (1986a). Instructions, multiple schedules, and extinction: Distinguishing rule-governed from schedule-controlled behavior. Journal of the Experimental Analysis of Behavior, 46, 137-147.

Hayes, S. C., Brownstein, A. J., Zettle, R. D., Rosenfarb, I. \& Korn, Z. (1986b). Rule governed behavior and sensitivity to changing consequences of responding. Journal of the Experimental Analysis of Behavior, 45, 237-256.

Hayes, S. C., Zettle, R. \& Rosenfarb, I. (1989). Rule-following. Em S. C. Hayes (Org.), Rule governed behavior: Cognition, contingencies, and intructional control (pp.191-220). New York: Plenum.

Joyce, J. H. \& Chase, P. N. (1990). Effects os response variability on the sensitivity of rule-governed behavior. Journal of the Experimental Analysis of Behavior, 54, 251-262.

LeFrancois, J. R., Chase, P. N. \& Joyce, J. H. (1988). The effects of variety of instructions on human fixed-interval performance. Journal of the Experimental Analysis of Behavior, 49, 383-393.

Lippman, L. G. \& Meyer, M. E. (1967). Fixed interval performance as related to instructions and to subject's verblizations of the contingency. Psychonomic Science, 8, 135-136.
Lowe, C. F. (1979). Determinants of human operant behaviour. Em M. D. Zeiler \& P. Harzem (Orgs.), Advances in analysis of behaviour (Vol. 1: Reinforcement and the organization of behaviour; pp.159-192). Chichester, England: Wiley.

Malott, R. W. (1989). The achievement of evasive goals: Control by rules describing contingencies that are not direct acting. Em S. C. Hayes (Org.), Rule governed behavior: Cognition, contingencies, and intructional control (pp. 269-322). New York: Plenum.

Matthews, B. A., Shimoff, E., Catania, A. C. \& Sagvolden, T. (1977). Uninstructed human responding: Sensitivity to ratio and interval contingencies. Journal of the Experimental Analysis of Bahavior, 27, 453-467.

Michael, R. L. \& Bernstein, D. J. (1991). Transient effects of acquisition history on generalization in a matching-to-sample task. Journal of the Experimental Analysis of Behavior, 56, 155-166.

Paracampo, C. C. P., Albuquerque, L. C. \& Fontes, J. C. S. (1993). Análise de algumas das variáveis responsáveis pela manutenção do seguimento de regras [Resumos]. Em Sociedade Brasileira para o Progresso da Ciência (Org.), Anais, $45^{a}$ Reunião Anual da Sociedade Brasileirapara o Progresso da Ciência (p. 984). Recife, PE: SBPC.

Paracampo, C. C. P., Souza, D. G., Matos, M. A. \& Albuquerque, L. C. (2001). Efeitos de mudanças em contingências de reforço sobre o comportamento verbal e o não-verbal. Acta Comportamentalia, 9, 31-55.

Parrott, L. J. (1987). Rule-governed behavior. Na implicit analysis of reference. Em S. Modgil \& C. Modgil (Orgs.), B. F. Skinner: Consensus and controversy (pp. 265276). Sussex: Falmer Press.

Perone, M., Galizio, M. \& Baron, A. (1988). The relevance of animal-based principles in the laboratory study of human operant conditioning. Em G. Davey \& C. Cullen (Orgs.), Human operant conditioning and behavior modification (pp.59-85). New York: Wiley \& Sons.

Shimoff, E., Catania, A. C. \& Matthews, B. A. (1981). Uninstructed human responding: Sensitivity of low-rate performance to schedule contingencies. Journal of the Experimental Analysis of Behavior, 36, 207-220.

Shimoff, E., Matthews, B. A. \& Catania, A. C. (1986). Human operant performance: Sensitivity and pseudosensitivity to contingencies. Journal of the Experimental Analysis of Behavior, 46, 149-157.

Sidman, M. (1960). Tactics of scientific research. Nova York: Basic Books.

Skinner, B. F. (1969). Contingencies of reinforcement: A theoretical analysis. New York: Appleton-Century-Crofts.

Skinner, B. F. (1974). About behaviorism. New York: Alfred A. Knopf.

Torgrud, L. J. \& Holborn, S. W. (1990). The effects of verbal performance descriptions on nonverbal operant responding. Journal of the Experimental Analysis of Behavior, 54, 273-291.

Weiner, H. (1970). Instructional control of human operant responding during extinction following fixed-ratio conditioning. Journal of the Experimental Analysis of Behavior, 13, 391-394.

Sobre os autores

José Guilherme Wady Santos é Mestre em Teoria e Pesquisa do Comportamento, Professor da Faculdade Ideal e Coordenador da Especialização em Gestão de Pessoas da "FAZ".

Carla Cristina Paiva Paracampo é Professora do Departamento de Psicologia Experimental da Universidade Federal do Pará e do Programa de Pós-Graduação em Teoria e Pesquisa do Comportamento da mesma instituição.

Luiz Carlos de Albuquerque é Professor do Departamento de Psicologia Experimental da Universidade Federal do Pará e Coordenador do Programa de Pós-Graduação em Teoria e Pesquisa do Comportamento da mesma instituição. 